\title{
A Longitudinal Study of the Association of Opioid Use with Change in Pain Interference and Functional Limitations in a Nationally Representative Cohort of Adults with Osteoarthritis in the United States
}

\author{
Drishti Shah - Xiaohui Zhao - Wenhui Wei - Kavita Gandhi • \\ Nilanjana Dwibedi · Lynn Webster · Usha Sambamoorthi
}

Received: October 18, 2019 / Published online: December 24, 2019

(C) The Author(s) 2019

\begin{abstract}
Introduction: Real-world data are sparse on longitudinal associations of opioid use with pain interference with activities (PIA) and daily function with osteoarthritis (OA) in the USA.

Methods: Data from the 2010-2015 Medical Expenditure Panel Surveys were analyzed for community-dwelling adults with OA. Opioid use patterns were defined as persistent, intermittent, and no use. Evaluated outcomes were a change in PIA and functional limitations (activities of daily living [ADL], instrumental ADL [IADL], social and work activities, and cognitive function). Multivariable regression analyses explored the association of persistent/
\end{abstract}

Enhanced Digital Features To view enhanced digital features for this article go to https://doi.org/10.6084/ m9.figshare.11353643.

D. Shah $\cdot$ X. Zhao $\cdot$ N. Dwibedi .

U. Sambamoorthi $(\square)$

Department of Pharmaceutical Systems and Policy,

West Virginia University School of Pharmacy,

Morgantown, WV, USA

e-mail: usambamoorthi@hsc.wvu.edu

W. Wei

Regeneron Pharmaceuticals, Tarrytown, NY, USA

K. Gandhi

Teva Pharmaceutical Industries, Frazer, PA, USA

L. Webster

PRA Health Sciences, Salt Lake City, UT, USA intermittent versus no opioid use with PIA and functional limitations. Results were weighted for the US population.

Results: Among 4172 patients (66.2\% female, $80.8 \%$ white, mean age 61.7 years), $62.1 \%$ reported no PIA change at follow-up, $17.9 \%$ worsened, and $20.0 \%$ improved. Although $51.0-93.1 \%$ of patients reported no functional limitations, $3.8-13.1 \%$ worsened (1.1-11.3\% improved). Relative to no opioid use, persistent users had higher odds of severe/extreme PIA (adjusted odds ratio [AOR], 2.91; 95\% confidence interval $[\mathrm{CI}], 1.95-4.32 ; P<0.001)$ and moderate PIA (AOR, 2.04; 95\% CI, 1.31-3.20; $P<0.01)$ at follow-up. For patients with baseline functional limitations, persistent opioid users were more likely to report physical and work limitations at follow-up (both $P<0.05$ ). For patients without baseline functional limitations, persistent opioid users had higher odds than those without use of reporting IADL, physical, social, and cognitive limitations at follow-up (all $P<0.05$ ); intermittent users were more likely to report physical and social limitations (both $P<0.05$ ).

Conclusions: Persistent opioid use for pain in patients with OA appeared to be associated with poorer PIA and functional outcomes, regardless of baseline functional status. These findings highlight the importance of patient-reported outcomes for opioid benefit-risk assessment and suggest the need for alternative analgesic approaches. 
Keywords: Functional limitations; Medical expenditure panel survey; Pain interference with activities; Opioids; Osteoarthritis

\section{Key Summary Points}

Why carry out this study?

Opioids are treatment options for patients with osteoarthritis pain, and although such use may be of potential benefit in managing osteoarthritis pain, there is a need for understanding functional outcomes in these patients

There are few data from the real-world clinical setting on the association of opioid use with pain interference with activities and daily function among adults with osteoarthritis

This exploratory, retrospective analysis evaluated the longitudinal association of opioid use with pain interference with daily activities and functional limitations among adults with osteoarthritis in the USA

What was learned from the study?

Regardless of baseline functional status, persistent opioid use in adults with osteoarthritis pain may be associated with poorer functional outcomes

These results highlight the importance of evaluating functional outcomes when assessing the potential benefits and risks of opioid use in patients with osteoarthritis and pain, and suggest a need for alternative pain management strategies

\section{INTRODUCTION}

Osteoarthritis (OA), the most common form of arthritis, is one of the leading causes of global disability [1]. In the USA, OA affects approximately 31 million individuals, and the prevalence appears to be increasing $[2,3]$. Pain is a primary symptom of OA that impairs function and limits daily activities [4]. The presence of pain increases the burden of OA by resulting in greater interference with daily activities, including reductions in work productivity and increasing healthcare resource utilization and associated costs relative to those without pain [5].

In the absence of disease-modifying therapies, the management of OA is symptomatically driven, with the reduction of pain and the maintenance of function being the primary goals of long-term treatment $[6,7]$. Although nonpharmacologic therapies (weight reduction, exercise, physical therapy) are generally recommended as part of an initial disease management strategy, nonsteroidal antiinflammatory drugs (NSAIDs) are considered a first-line pharmacologic therapy for use in the short-term management of OA in specific subsets of patients (i.e., those not at high risk for gastrointestinal or cardiovascular events) [6-8]. However, opioids have been reported to be prescribed in as many as $40 \%$ of patients with OA [9-12], although these drugs are generally recommended in patients with OA specifically for intense or refractory pain and for those who do not respond to first-line treatment or have contraindications to other analgesics [6-8]. Furthermore, guidelines for the use of opioids in the management of chronic noncancer pain recommend that regimens be individualized and also require that the prescribing physician provide an evaluation of the risks and benefits [13].

Although opioids may be prescribed to provide symptomatic pain relief and subsequent improved function, there is limited evidence on the relationship of opioid use and daily function in patients with OA in the real-world setting. A previous cross-sectional study reported prescription opioid use in nearly one-third $(30.3 \%)$ of individuals with OA and showed that opioid users had a higher disease burden compared with those without opioid use, even after adjusting for relevant demographic and clinical variables [14]. Furthermore, a study on the association of opioids and pain interference 
with activities (PIA) among individuals with OA showed that regardless of the level of PIA, opioid use increased the likelihood of specific categories of healthcare resource utilization including hospitalizations and emergency room visits, and was associated with higher costs relative to those not using opioids [15]. The purpose of the current study is to explore longitudinal assessment of the association of opioid use with PIA and functional limitations among adults with OA in the USA.

\section{METHODS}

\section{Data Source and Study Sample}

The data source for this exploratory, retrospective, longitudinal study was the Medical Expenditure Panel Survey (MEPS) for 2010-2015. MEPS is sponsored by the Agency for Healthcare Research and Quality and is a nationally representative annual survey of the civilian noninstitutionalized population in the USA $[16,17]$. Data collected from families and individuals during the survey included demographic characteristics; socioeconomic status; medical conditions; and health status, such as functional and activity limitations; as well as health-related quality of life; use of medical services; medical expenditures; access to care; and health insurance coverage. Because this study used secondary data from MEPS, which is a publicly available database, all ethics approvals and consent to participate were waived by the institutional review board at West Virginia University.

These data are collected in interview panels, with each panel following the same individuals for two consecutive years. A total of five rounds of data (interviews) are collected over the course of each 2-year panel (Fig. 1). This panel design enables longitudinal assessment of outcomes for each participant over a 2-year period. The current analysis was based on panels for 2010-2011, 2011-2012, 2012-2013, 2013-2014, and 2014-2015, corresponding to panels 15, 16, 17, 18, and 19, respectively, in the MEPS database.
The criteria used for identification of the evaluated population was the same as that in a previously published study on the association of opioid use and PIA with economic outcomes [15]. For inclusion in the analysis, adults (aged at least 18 years old) with OA were identified in the MEPS database for panels 15-19 from queries related to whether the respondent had "OA" in the full-year consolidated files or medical care encounters recorded in the medical condition files using the Clinical Classification Code (CCC) identifier 203. This CCC identifier matches to the International Classification of Diseases, Ninth Revision, Clinical Modification (ICD-9-CM) codes 715.xx (osteoarthrosis and allied disorders). The CCC code of 203 also includes the ICD-9 code V134 (personal history of arthritis). This code was considered relevant because including this code could help identify patients with OA who did not seek healthcare services for $\mathrm{OA}$ in the year surveyed. Individuals reporting any cancer (CCC 11-44) were excluded.

\section{Outcomes}

The outcomes were PIA and functional limitations during the follow-up period (Fig. 1). PIA, which was collected in rounds 2 (baseline) and 4 (follow-up) in each panel, was derived from the 12-item Short Form Health Survey version 2 (SF-12 v2) health survey [18]: "During the past 4 weeks, how much did pain interfere with your normal work (including both work outside the home and housework)?" with response options of "not at all," "a little bit," "moderately," "quite a bit," and "extremely." In the current analysis, these responses were recategorized as no/mild (responses of "not at all/a little bit"), moderate (response of "moderately"), and severe (responses of "quite a bit/extremely").

Changes in PIA groups were considered "better" if an individual moved from a higher to a lower PIA level during follow-up, "worse" if the movement was from a lower to a higher PIA level during follow-up, and "no change" if PIA levels were the same at both baseline and follow-up. 


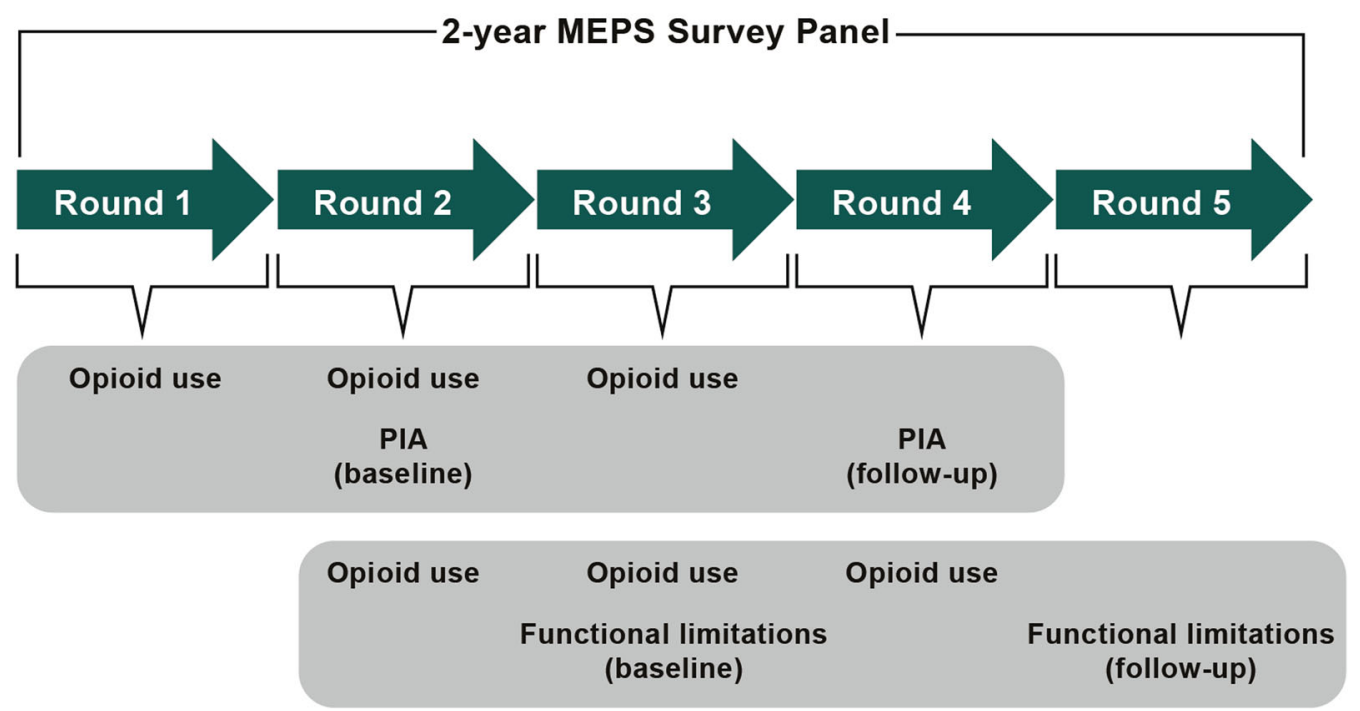

Fig. 1 Study design and data collection. MEPS Medical Expenditure Panel Survey, PIA pain interference with daily activities

Functional limitations, dichotomized as "Yes" or "No" and collected in rounds 3 (baseline) and 5 (follow-up) in each panel, included any limitations in activities of daily living (ADL), instrumental ADL (IADL), physical activities, social function, work, and cognitive function. Although ADL is defined as the basic activities required for daily function (e.g., bathing, eating, dressing, transferring, toileting, and walking) $[19,20]$, IADL are not required for daily functioning but contribute to maintaining an individual's independence (e.g., housework, preparing meals, taking medications, shopping, telephoning, and managing money) [21, 22]. Changes in functional limitations were defined by the following four categories: "better" if an individual with a limitation at baseline was not limited at follow-up, "worse" if there was a limitation at follow-up in an individual with no limitation at baseline, "same" if there were limitations at baseline and follow-up, and those with no limitations at both baseline and followup.

\section{Statistical Analysis}

Study participants reported their opioid use at every round and thus definitions of opioid use patterns needed to account for the manner in which data were collected. Thus, persistent use was defined when opioid use was reported in at least two consecutive rounds before the last round of follow-up (rounds 1-3 for PIA analysis and rounds 2-4 for functional limitation analysis), and intermittent opioid use defined when reported in any one of the rounds; no opioid use was defined if such use was not reported in any of the rounds.

Unadjusted differences across the opioid use patterns for observed longitudinal changes in PIA and functional limitations were evaluated using Rao-Scott chi-square tests. Multinomial logistic regressions were conducted to examine the association between opioid use patterns and PIA during follow-up. Stratified multivariable logistic regressions explored the association of persistent or intermittent use of opioids versus no use of opioids with follow-up functional status; the stratification variable was the baseline functional status (i.e., patients with or without functional limitations at baseline).

Adjustment for covariates in the regression models was based on Andersen's Behavioral Model of Health Services Use that included demographics, comorbidities, and health characteristics [23]. Specific variables included in this model were predisposing factors (age group, sex, race/ethnicity), enabling factors (marital 
status, educational level, employment status, economic status based on family income relative to the federal poverty line, health insurance coverage, prescription drug coverage), external environment (geographic region), need factors (number of comorbidities, depression and anxiety), and personal health practices (smoking status, body mass index, and physical activity [at least five times a week]). In the adjusted analyses, no opioid use with no/mild PIA was used as the reference group. The regression analyses also adjusted for the use of prescription NSAIDs, which was determined in every round and defined in a manner similar to that for opioid use (i.e., persistent, intermittent, and no use). Regression results are expressed as adjusted odds ratios (AOR) and their 95\% confidence intervals (CIs).

All analyses account for the complex survey design of MEPS and were conducted using SAS version 9.4 (SAS Institute Inc., Cary, NC). Weights specifically assigned for longitudinal data files (LONGWT) were used to account for the longitudinal design. All reported results were weighted to be nationally representative.

\section{RESULTS}

\section{Population Characteristics}

A total of 4172 adults with OA were identified for inclusion in the analysis; the majority were female $(66.2 \%)$, white $(80.8 \%)$, and aged at least 50 years old $(82.8 \%)$, with a high prevalence of overweight (32.4\%) and obesity (40.4\%; Table 1). Approximately one-third of adults had opioid use in rounds $1-3$ (35.4\%) and 2-4 (32.7\%), with intermittent use more prevalent than persistent use (Fig. 2). Prescription NSAID use was $25.3 \%$ in rounds $1-3$ and $24.0 \%$ in rounds $2-4$, with a slightly greater prevalence of intermittent use (Fig. 2).

\section{Unadjusted Analysis}

Changes from baseline to follow-up in PIA and functional status are presented in Table 2, which also shows the unadjusted differences
Table 1 Characteristics in baseline year among adults with osteoarthritis, using pooled data from Medical Expenditure Panel Survey for the years 2010-2015 $(N=4172)$

\begin{tabular}{|c|c|}
\hline Variable & $\begin{array}{l}\text { Number (weighted \%) } \\
\text { of patients }\end{array}$ \\
\hline Female & $2858(66.2)$ \\
\hline \multicolumn{2}{|l|}{ Race/ethnicity } \\
\hline White & $2606(80.8)$ \\
\hline African American & $754(8.2)$ \\
\hline Latino & $540(6.1)$ \\
\hline Other & $272(4.8)$ \\
\hline \multicolumn{2}{|l|}{ Age distribution, years } \\
\hline $18-39$ & $279(6.3)$ \\
\hline $40-49$ & $496(10.9)$ \\
\hline $50-64$ & $1699(41.0)$ \\
\hline $65-74$ & $946(23.0)$ \\
\hline$\geq 75$ & $752(18.8)$ \\
\hline \multicolumn{2}{|l|}{ Marital status } \\
\hline Married & $2137(56.3)$ \\
\hline Widow & $641(14.3)$ \\
\hline Separated/divorced & $919(20.2)$ \\
\hline Never married & $475(9.2)$ \\
\hline \multicolumn{2}{|l|}{ Education } \\
\hline Less than high school & $554(9.4)$ \\
\hline High school & $951(21.3)$ \\
\hline More than high school & $1777(48.3)$ \\
\hline Missing & $890(21.0)$ \\
\hline Employed & $1733(44.7)$ \\
\hline \multicolumn{2}{|l|}{ Income } \\
\hline Poor & $712(12.3)$ \\
\hline Near poor & $938(19.1)$ \\
\hline Middle income & $1153(27.3)$ \\
\hline High income & $1369(41.2)$ \\
\hline \multicolumn{2}{|l|}{ Health insurance coverage } \\
\hline Private & $2382(65.1)$ \\
\hline
\end{tabular}


Table 1 continued

\begin{tabular}{|c|c|}
\hline Variable & $\begin{array}{l}\text { Number (weighted \%) } \\
\text { of patients }\end{array}$ \\
\hline Public & $1467(29.4)$ \\
\hline Uninsured & $323(5.5)$ \\
\hline Prescription drug coverage & $1577(42.8)$ \\
\hline Usual source of care & $3767(90.9)$ \\
\hline \multicolumn{2}{|l|}{ Number of chronic conditions } \\
\hline 0 & $944(23.7)$ \\
\hline 1 & $1052(24.6)$ \\
\hline 2 & $893(21.4)$ \\
\hline$\geq 3$ & $1283(30.3)$ \\
\hline \multicolumn{2}{|l|}{ Depression/anxiety } \\
\hline No depression or anxiety & $2959(69.9)$ \\
\hline Depression only & $556(13.3)$ \\
\hline Anxiety only & $390(9.9)$ \\
\hline Depression and anxiety & $267(6.8)$ \\
\hline \multicolumn{2}{|l|}{ Body mass index } \\
\hline Under/normal weight & $993(25.3)$ \\
\hline Overweight & $1329(32.4)$ \\
\hline Obese & $1773(40.4)$ \\
\hline Missing & $77(1.9)$ \\
\hline Current smoker & $648(14.5)$ \\
\hline \multicolumn{2}{|l|}{ Region } \\
\hline Northeast & $740(19.6)$ \\
\hline Midwest & $970(24.6)$ \\
\hline South & $1592(37.2)$ \\
\hline West & $870(18.5)$ \\
\hline
\end{tabular}

across the opioid use patterns. In the total population, $62.1 \%$ of patients had no PIA change, $17.9 \%$ worsened, and $20.0 \%$ improved. Although the majority of patients (51.0-93.1\%) reported no limitations in each functional category, when changes did occur, patients more frequently reported that their functional status worsened (3.8-13.1\%) than improved (1.1-11.3\%). There was no statistically significant difference across opioid use patterns for changes in PIA, but the changes in all functional categories were statistically significant across the opioid use patterns (all $P<0.001$ ) (Table 2). The proportion of adults who worsened was significantly higher among those with either persistent or intermittent opioid use compared with no opioid use, specifically for functional limitations in physical, social, and work activities.

\section{Adjusted Analyses}

Multivariable regression analyses that adjusted for baseline sociodemographic and clinical characteristics, prescription NSAID use, and baseline PIA showed that relative to those without opioid use, persistent opioid users were approximately three times more likely to report extreme/severe PIA (AOR, 2.91; 95\% CI, $1.95-4.32 ; P<0.001)$ and two times more likely to report moderate PIA (AOR, 2.04; 95\% CI, $1.31-3.20 ; P<0.001)$ instead of mild/no PIA at follow-up (Fig. 3). No statistically significant differences were observed between intermittent and no opioid use for the changes in PIA at follow-up (Fig. 3).

Patients who had functional limitations at baseline and persistent opioid use were statistically significantly more likely at follow-up to report work (AOR, 1.90; 95\% CI, 1.06-3.39) and physical limitations (AOR, 1.63; 95\% CI, 1.07-2.49) compared with those who had no opioid use (both $P<0.05$; Fig. 4 a); no significant differences were observed for social or cognitive limitations. Among intermittent opioid users who had functional limitations at baseline, the odds of social and cognitive limitations at follow-up were significantly and substantially lower than among those with no opioid use; AOR, 0.35 (95\% CI, 0.17-0.72) and AOR, 0.28 (95\% CI, 0.13-0.62), respectively (both $P<0.01$ ). In this subpopulation of those with functional limitations at baseline, ADL and IADL could not be determined because of the 


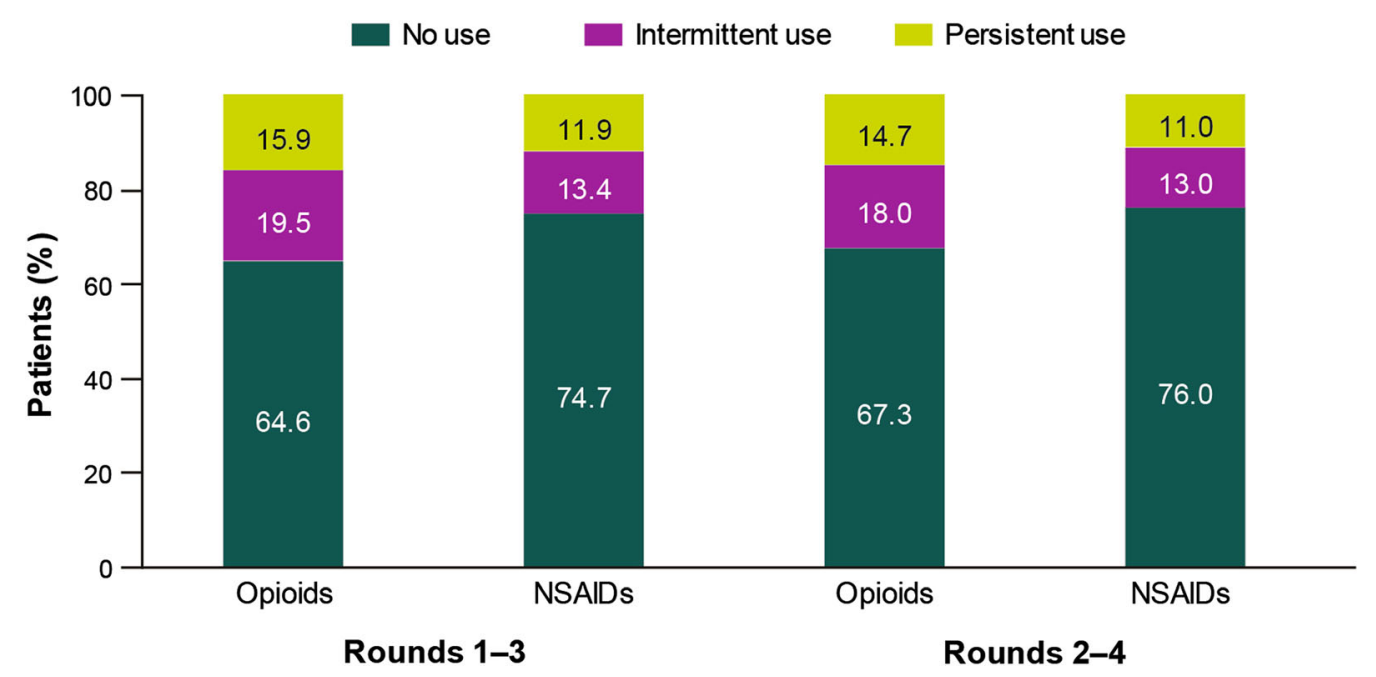

Fig. 2 Patterns of use of opioids and prescription NSAIDs. NSAID nonsteroidal anti-inflammatory drug

small sample sizes, which resulted in a high relative standard error (>30\%).

For patients without baseline functional limitations (Fig. 4b), persistent opioid users were significantly more likely to report cognitive, social, and physical limitations at followup, as well as limitations in performing IADL compared with those with no opioid use. The highest adjusted odds were for physical limitations (AOR, 2.39; 95\% CI, 1.50-3.80; $P<0.001$ ), followed by cognitive limitations (AOR, 1.80; 95\% CI, 1.13-2.84; $P<0.05)$. Similarly, intermittent opioid users compared with those with no opioid use were more likely to report limitations in physical (AOR, 1.52; 95\% CI, 1.02-2.26; $P<0.05$ ) and social (AOR, 1.44; 95\% CI, 1.00-2.07; $P<0.05$ ) functions (Fig. 4b).

\section{DISCUSSION}

$\mathrm{OA}$ is characterized by pain and functional impairment and, thus, it is important that therapeutic strategies effectively reduce pain and maintain daily function-outcomes that are the primary goals in the management of patients with OA [6, 7]. However, accumulating evidence has raised uncertainties about whether the small benefits of opioids in some patients outweigh their risks as a strategy for meeting these goals [14, 24, 25]. This current study provides further evidence from real-world clinical settings that opioids, either with specific patterns of use or in certain subpopulations, may be associated with poorer outcomes with regard to maintaining function.

In particular, persistent opioid use appeared to be associated with greater negative effects than intermittent opioid use on PIA and function for a subset of patients. After adjusting for relevant demographic and clinical variables, we can interpret the statistically significant AORs of 2.91 and 2.04 for severe/extreme and moderate PIA, respectively, as approximate two- and threefold greater odds that patients with persistent use of opioids, relative to those without opioid use, report these levels of PIA instead of no PIA. Although there are limited data on the long-term use of opioids, these results are consistent with a recent study suggesting no additional benefits of long-term opioid use compared with other analgesic classes for painrelated function in a subset of patients with OA [25]. Indeed, regardless of baseline functional status in the current study, persistent opioid use was associated with a high odds of limitations in functional categories, specifically physical limitations during follow-up, compared with those with no opioid use. There are many factors that could potentially contribute to these functional limitations, such as higher disease severity, obesity, and the ability of patients to cope with and adapt to limitations. It is also likely that some of these limitations may be 
Table 2 Changes in functional status and PIA from baseline to follow-up

\begin{tabular}{|c|c|c|c|c|c|}
\hline \multirow[t]{2}{*}{ Outcome } & \multicolumn{4}{|c|}{ Number (\%) of patients } & \multirow[t]{2}{*}{$P^{\mathbf{a}}$} \\
\hline & Overall & No opioid use & Intermittent opioid use & Persistent opioid use & \\
\hline \multicolumn{6}{|l|}{ PIA $(n=4172)^{\mathrm{b}}$} \\
\hline Same & $2544(62.1)$ & $1718(63.3)$ & $436(58.2)$ & $390(61.4)$ & \\
\hline Better & $879(20.0)$ & $553(18.4)$ & $189(24.4)$ & $137(22.0)$ & \\
\hline Worse & $749(17.9)$ & $521(18.3)$ & $121(17.4)$ & $107(16.6)$ & NS \\
\hline \multicolumn{6}{|l|}{ Functional limitations ${ }^{c}$} \\
\hline $\mathrm{ADL}(n=4102)$ & & & & & $<0.001$ \\
\hline No limitation & $3771(93.1)$ & $2501(95.5)$ & $708(92.0)$ & $562(84.9)$ & \\
\hline Same & $94(1.9)$ & $42(1.3)$ & $15(2.1)$ & $37(4.1)$ & \\
\hline Better & $63(1.1)$ & $21(0.5)$ & $16(1.8)$ & $26(3.1)$ & \\
\hline Worse & $174(3.8)$ & $87(2.7)$ & $33(4.1)$ & $54(8.0)$ & \\
\hline $\operatorname{IADL}(n=4101)$ & & & & & $<0.001$ \\
\hline No limitation & $3509(87.6)$ & $2368(91.1)$ & $650(85.9)$ & $491(75.6)$ & \\
\hline Same & $193(4.1)$ & $102(3.5)$ & $39(4.7)$ & $52(6.0)$ & \\
\hline Better & $146(2.8)$ & $61(1.7)$ & $36(3.8)$ & $49(5.8)$ & \\
\hline Worse & $253(5.4)$ & $119(3.7)$ & $47(5.6)$ & $87(12.5)$ & \\
\hline Physical $(n=4098)$ & & & & & $<0.001$ \\
\hline No limitation & $2055(51.0)$ & $1583(60.7)$ & $333(42.5)$ & $139(22.4)$ & \\
\hline Same & $1037(24.6)$ & $471(17.5)$ & $226(29.1)$ & $340(48.0)$ & \\
\hline Better & $484(11.3)$ & $290(10.4)$ & $98(12.6)$ & $96(13.5)$ & \\
\hline Worse & $522(13.1)$ & $303(11.5)$ & $116(15.7)$ & $103(16.1)$ & \\
\hline Social $(n=4094)$ & & & & & $<0.001$ \\
\hline No limitation & $3166(78.1)$ & 2239 (84.9) & $554(72.4)$ & $373(57.1)$ & \\
\hline Same & $305(7.0)$ & $118(4.4)$ & $56(6.5)$ & $131(18.1)$ & \\
\hline Better & $228(5.1)$ & $103(3.5)$ & $65(7.2)$ & $60(8.8)$ & \\
\hline Worse & $395(9.9)$ & $189(7.1)$ & $94(14.0)$ & $112(15.9)$ & \\
\hline Work $(n=4097)$ & & & & & $<0.001$ \\
\hline No limitation & $2656(67.1)$ & $1958(75.5)$ & $445(59.8)$ & $253(41.7)$ & \\
\hline Same & $731(16.6)$ & $299(10.9)$ & $163(19.6)$ & $269(36.5)$ & \\
\hline Better & $271(5.8)$ & $155(5.2)$ & $70(7.7)$ & $46(6.4)$ & \\
\hline Worse & $439(10.4)$ & $239(8.5)$ & $92(12.9)$ & $108(15.4)$ & \\
\hline
\end{tabular}


Table 2 continued

\begin{tabular}{llclll}
\hline Outcome & \multicolumn{2}{l}{ Number (\%) of patients } & & $P^{\mathbf{a}}$ \\
\cline { 2 - 5 } & Overall & No opioid use & Intermittent opioid use & Persistent opioid use & \\
\hline Cognitive $(n=4097)$ & & & & & $<0.001$ \\
No limitation & $3388(85.1)$ & $2311(89.5)$ & $633(83.2)$ & $444(69.5)$ & \\
Same & $266(5.4)$ & $122(4.0)$ & $42(4.5)$ & $102(12.3)$ \\
Better & $185(3.8)$ & $87(2.8)$ & $45(4.8)$ & $53(6.5)$ \\
Worse & $258(5.7)$ & $126(3.6)$ & $52(7.4)$ & $80(11.7)$ & \\
\hline
\end{tabular}

$A D L$ activities of daily living, $I A D L$ instrumental activities of daily living, $N S$ not significant, PIA pain interference with activities

${ }^{a}$ Across the three opioid use patterns

b From round 2 to round 4, with changes defined as "better" if PIA level was higher at follow-up relative to baseline; "worse" if PIA level was lower at follow-up than at baseline; and "no change" if PIA levels were the same at baseline and follow-up c From round 3 to round 5, with changes defined by 4 categories: "better" if an individual with a limitation at baseline was not limited at follow-up; "worse" if a limitation at follow-up was observed in an individual with no limitation at baseline; "same" if their limitations were present at baseline and follow-up; and those with no limitations at both baseline and followup

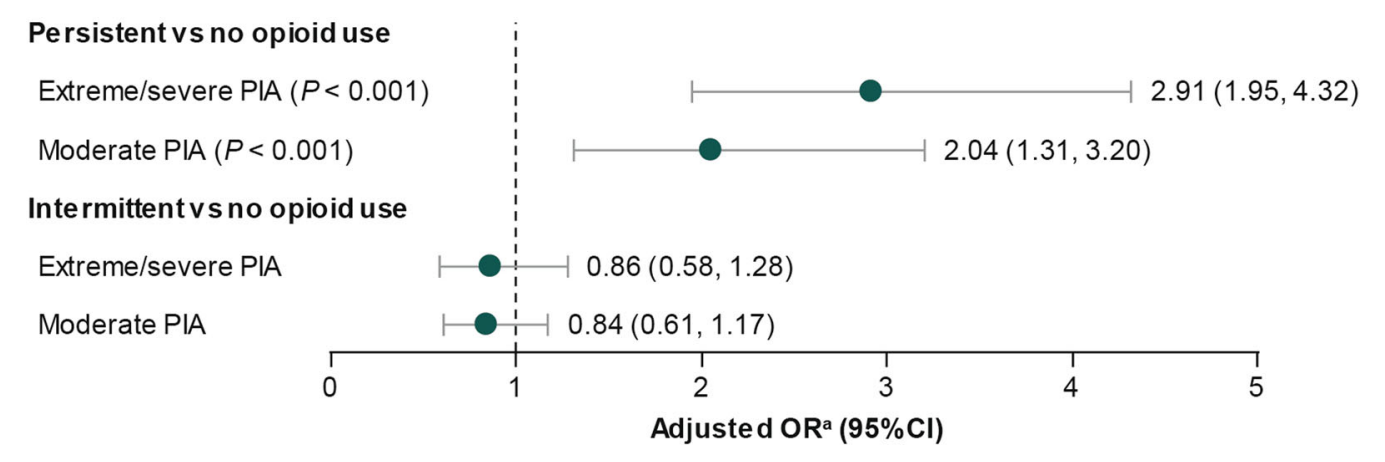

Fig. 3 Adjusted OR for PIA at follow-up relative to no/ mild PIA (referent) by opioid use pattern. ${ }^{\text {a Adjusted for }}$ baseline sociodemographic and clinical characteristics,

attributed, at least in part, to the side effects of long-term opioid use, which can be exacerbated in an older population [26] or in medically complex patients, such as those characterized by comorbid conditions and the use of polypharmacy [27], both of which are common among patients with OA [28]. Patients treated chronically with opioids may also become refractory to therapy, contributing to functional limitations. The generally greater association of PIA with physical limitations is considered as a prescription use of nonsteroidal anti-inflammatory drugs, and baseline PIA. $C I$ confidence interval, $O R$ odds ratio, PIA pain interference with activities

proximal symptom of pain and is supported by previous studies in patients with OA, where increasing or unresolved pain was a predictor of functional decline, especially related to physical activities [29-31].

In contrast to persistent opioid use, intermittent opioid use appeared to be associated with a reduced likelihood of reporting social and cognitive limitations compared with no opioid use among those with such limitations at baseline; for those without baseline functional 


\section{(a) Functional limitation at baseline ${ }^{a}$}

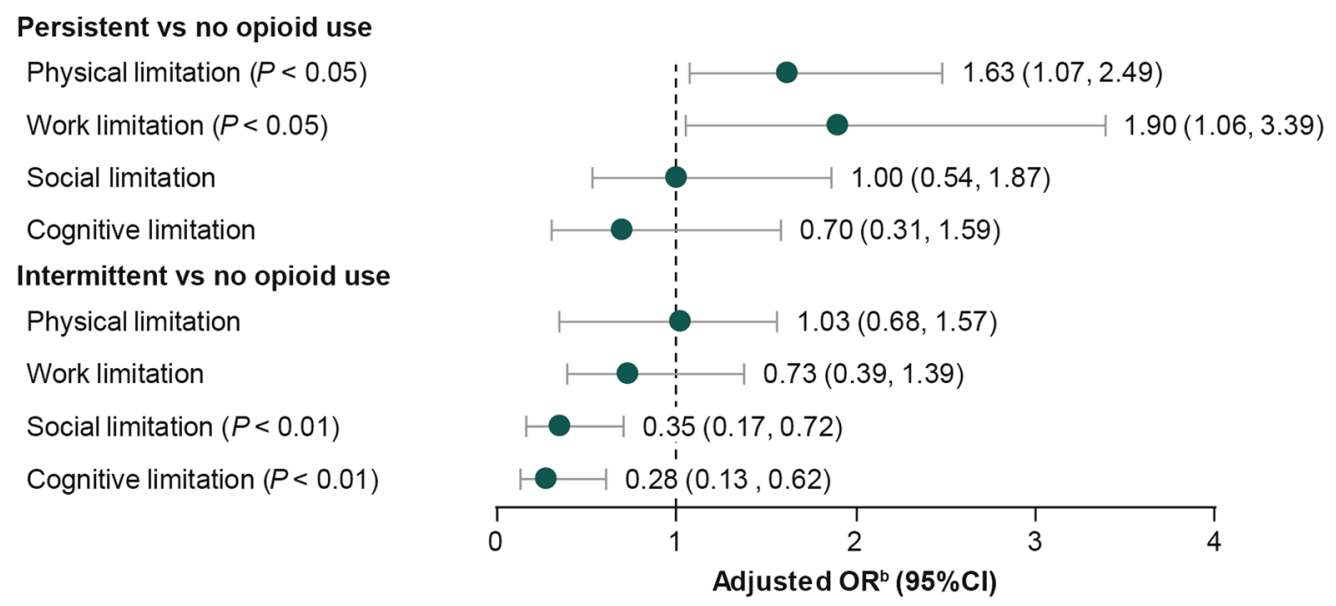

(b) No functional limitation at baseline
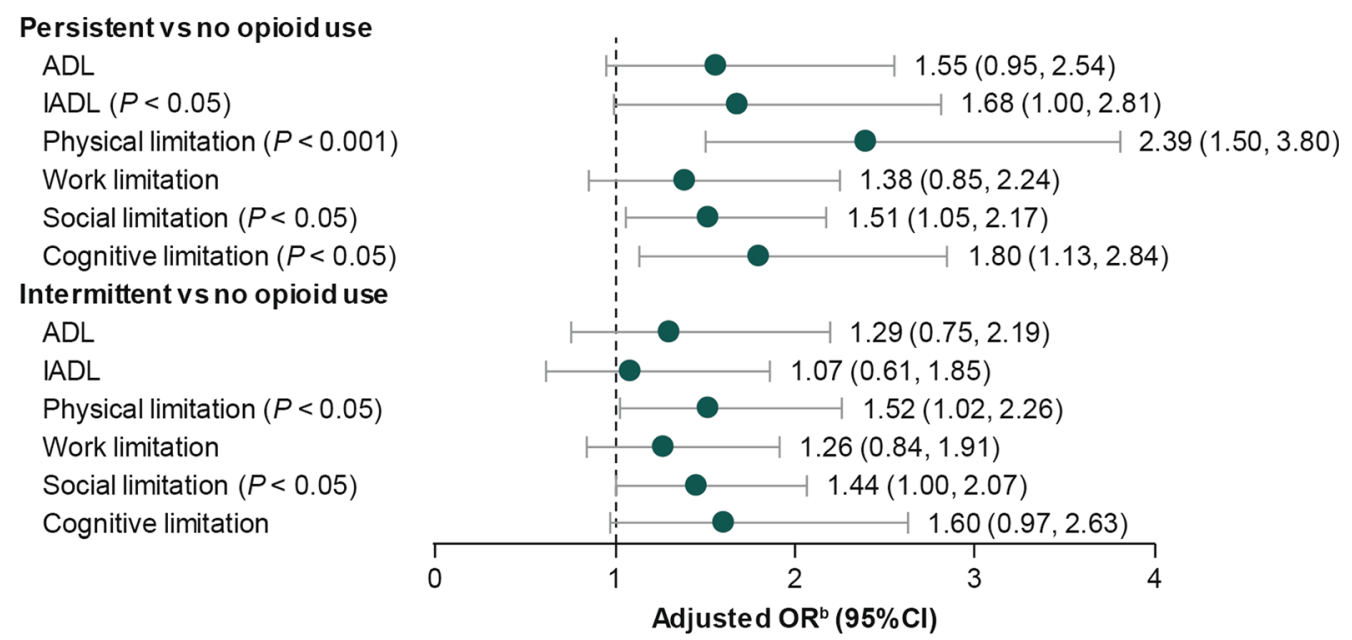

Fig. 4 Adjusted OR for functional limitations at followup by opioid use pattern among patients with osteoarthritis. ${ }^{a}$ Sample sizes were too small to evaluate ADL and IADL among patients with functional limitations at baseline. ${ }^{\mathrm{b}}$ Adjusted for baseline sociodemographic and

clinical characteristics, prescription use of nonsteroidal anti-inflammatory drugs, and baseline pain interference with daily activities. $A D L$ activities of daily living, $C I$ confidence interval, $L A D L$ instrumental activities of daily living, $O R$ odds ratio

limitations, intermittent opioid use was still associated with a higher likelihood of patients reporting physical and social limitations. These results suggest that intermittent opioid use may be associated with reduced PIA and improved functional status in certain subgroups and, therefore, may be worth evaluating as a potential treatment strategy for managing OA pain in future studies in conjunction with individual patient and clinical characteristics. These findings must also be interpreted with caution because intermittent opioid users with baseline functional limitations may have likely adapted to their limitations and other unobserved factors, such as disease severity, pain self-efficacy, and use of complementary or alternative therapies, which may also have contributed to the results. Therefore, because this study was not designed to explore the reasons for the observed limitations associated with opioid use, 
additional studies are warranted to confirm these observations and characterize factors that may be associated with greater functional limitations among patients with OA who are using opioids.

A major strength of this study is that evaluated outcomes incorporated PIA and aspects of daily activities, such as social and cognitive function, which may be impacted differentially by pain and patterns of treatment. Although the outcomes measures used have not been specifically validated for such an analysis, this study was designed as an initial exploration of the associations between opioid use and daily function in patients with OA. Additional strengths include the fact that the analysis adjusted for a comprehensive set of variables that may be associated with the evaluated outcomes and that the study sample is nationally representative of the US population.

Despite adjustment for a range of variables, an important study limitation is that unobservable factors may exist that could account for some of the differences in outcomes, such as those previously noted with regard to disease severity, no or limited response with long-term use of opioids, length of opioid use prior to baseline, and the use of complementary/alternative therapies. Other limitations include the use of self-reported data, which may result in potential recall and social desirability bias. In addition, several limitations are specifically associated with the collection of the opioid data, including the fact that opioid use was selfreported and collected only at the round level, the actual opioids used were not identified, the sample size was not robust enough to further differentiate short-acting opioids from longacting opioids, and there was insufficient information to calculate dosage and dosing frequency. The inability to incorporate information on strength and quantity of opioid medications while defining persistent and intermittent opioid use is another limitation, which resulted because there was a substantial amount of missing information (approximately $30 \%)$ for these variables in the MEPS database. The definition of long-term or persistent opioid use varies in the existing literature from 60 to 180 days or more [32-34]. Any two consecutive
MEPS rounds are approximately 4-6 months apart; therefore, adults reporting opioid use in nonconsecutive rounds may have long-term use. The fact that PIA and functional limitations were not measured in the same rounds of the survey is another limitation, as is the lack of control for unobserved patient or disease characteristics that may potentially affect evaluated outcomes. In this regard, MEPS does not collect information on over-the-counter medications; the use of over-the-counter medications such as NSAIDs, as well as other treatment modalities, could impact an individual's pain level and functional ability. In addition, MEPS does not include institutionalized individuals or individuals living with undiagnosed $\mathrm{OA}$, potentially reducing the generalizability of the results. Finally, analyses such as these are limited by the inability to link opioid prescriptions to the condition of interest. Thus, these findings must be interpreted as exploratory and associative rather than causal.

\section{CONCLUSIONS}

This real-world study suggests that persistent opioid use in adults with OA pain may be associated with poorer functional outcomes compared with OA pain without persistent opioid use. At follow-up, patients with persistent opioid use, especially those who did not have functional limitations at baseline, were more likely than patients without persistent opioid use to report limitations in daily function, especially related to physical activities. Persistent opioid use was also associated with a higher likelihood of moderate or severe/extreme PIA compared with no opioid use. Although opioids may be of benefit in some patients, these findings highlight the importance of evaluating the benefits and risks of opioids by the prescribing physician on an ongoing, case-by-case basis, taking into consideration patient characteristics and observed outcomes. Additionally, these findings suggest an unmet need for an alternative approach to pain management in a subgroup of patients with $\mathrm{OA}$ who use opioids. Future prospective studies are needed to validate these findings. 


\section{ACKNOWLEDGEMENTS}

Funding. For this study, the journal's Rapid Service and Open Access Fees were funded by Regeneron Pharmaceuticals, Inc. and Teva Pharmaceutical Industries, Ltd.

Medical Writing, Editorial, and Other Assistance. The authors acknowledge E. Jay Bienen, PhD, for medical writing support and StemScientific (an Ashfield Company, part of UDG Healthcare plc) for editorial support during development of this manuscript. Support for this assistance was funded by Regeneron Pharmaceuticals, Inc. and Teva Pharmaceutical Industries, Ltd.

Authorship. All named authors meet the International Committee of Medical Journal Editors (ICMJE) criteria for authorship for this article, take responsibility for the integrity of the work as a whole, and have given their approval for this version to be published.

Prior Presentation. Some data included in this manuscript were presented at the 2018 PAINWeek congress.

Disclosures. Drishti Shah, Xiaohui Zhao, Nilanjana Dwibedi, and Usha Sambamoorthi are paid consultants for Regeneron Pharmaceuticals, Inc. and Teva Pharmaceuticals Industries. Wenhui Wei is an employee and stockholder of Regeneron Pharmaceuticals, Inc. Lynn Webster has served on advisory boards for Charleston Laboratories, Depomed, and Inspirion, and has served as a consultant to Alcobra, Bonti, Charleston Laboratories, Eaglet, Depomed, Indivior, Insys, Kempharm, Pernix, Pain Therapeutics, Shionogi, Daiichi Sankyo, Trevena, Trevi, and Teva Pharmaceutical Industries. Kavita Gandhi was an employee of Teva Pharmaceutical Industries at the time of this study, and is currently an employee of Pfizer Inc.

Compliance with Ethics Guidelines. The current study used secondary data from the Medical Expenditures Panel Survey, a publicly available database. As such, all ethics approvals and consent to participate were waived by the institutional review board at West Virginia University.

Data Availability. The data used to support the findings of this study are from MEPS Household Component public use data files, which can be downloaded from https://meps. ahrq.gov/mepsweb/data_stats/download_data_ files.jsp.

Open Access. This article is licensed under a Creative Commons Attribution-NonCommercial 4.0 International License, which permits any non-commercial use, sharing, adaptation, distribution and reproduction in any medium or format, as long as you give appropriate credit to the original author(s) and the source, provide a link to the Creative Commons licence, and indicate if changes were made. The images or other third party material in this article are included in the article's Creative Commons licence, unless indicated otherwise in a credit line to the material. If material is not included in the article's Creative Commons licence and your intended use is not permitted by statutory regulation or exceeds the permitted use, you will need to obtain permission directly from the copyright holder. To view a copy of this licence, visit http://creativecommons.org/licenses/bync/4.0/.

\section{REFERENCES}

1. Smith E, Hoy DG, Cross M, et al. The global burden of other musculoskeletal disorders: estimates from the Global Burden of Disease 2010 study. Ann Rheum Dis. 2014;73(8):1462-9.

2. Cisternas MG, Murphy L, Sacks JJ, et al. Alternative methods for defining osteoarthritis and the impact on estimating prevalence in a US population-based survey. Arthritis Care Res (Hoboken). 2016;68(5): 574-80.

3. Park J, Mendy A, Vieira ER. Various types of arthritis in the United States: prevalence and age-related trends from 1999 to 2014. Am J Public Health. 2018;108(2):256-8.

4. Neogi T. The epidemiology and impact of pain in osteoarthritis. Osteoarthr Cartil. 2013;21(9): 1145-53. 
5. daCosta DiBonaventura M, Gupta S, McDonald M, Sadosky A. Evaluating the health and economic impact of osteoarthritis pain in the workforce: results from the National Health and Wellness Survey. BMC Musculoskelet Disord. 2011;12:83.

6. Zhang W, Moskowitz RW, Nuki G, et al. OARSI recommendations for the management of hip and knee osteoarthritis, Part II: OARSI evidence-based, expert consensus guidelines. Osteoarthr Cartil. 2008;16(2):137-62.

7. Hochberg MC, Altman RD, April KT, et al. American College of Rheumatology 2012 recommendations for the use of nonpharmacologic and pharmacologic therapies in osteoarthritis of the hand, hip, and knee. Arthritis Care Res (Hoboken). 2012;64(4): 465-74.

8. McAlindon TE, Bannuru RR, Sullivan MC, et al. OARSI guidelines for the non-surgical management of knee osteoarthritis. Osteoarthr Cartil. 2014;22(3):363-88.

9. Berger A, Bozic K, Stacey B, et al. Patterns of pharmacotherapy and health care utilization and costs prior to total hip or total knee replacement in patients with osteoarthritis. Arthritis Rheum. 2011;63(8):2268-75.

10. Gore M, Tai KS, Sadosky A, Leslie D, Stacey BR. Use and costs of prescription medications and alternative treatments in patients with osteoarthritis and chronic low back pain in community-based settings. Pain Pract. 2012;12(7):550-60.

11. Wright EA, Katz JN, Abrams S, Solomon DH, Losina E. Trends in prescription of opioids from 2003-2009 in persons with knee osteoarthritis. Arthritis Care Res (Hoboken). 2014;66(10):1489-95.

12. DeMik DE, Bedard NA, Dowdle SB, et al. Are we still prescribing opioids for osteoarthritis? J Arthroplasty. 2017;32(12):3578-82.

13. Dowell D, Haegerich TM, Chou R. CDC guideline for prescribing opioids for chronic pain-United States, 2016. JAMA. 2016;315(15):1624-45.

14. Shah D, Zhao X, Gandhi K, et al. Opioid use, pain interference with activities, and their associated burden in a nationally representative sample of adults with osteoarthritis in the United States (US): results of a retrospective, cross-sectional analysis [abstract 276]. Pain Med. 2018;19(4):893.

15. Zhao X, Shah D, Gandhi K, et al. The association of pain interference and opioid use with healthcare utilization and costs, and wage loss among adults with osteoarthritis in the United States. J Med Econ. 2019;22(11):1192-201.
16. Cohen JW, Cohen SB, Banthin JS. The Medical Expenditure Panel Survey: a national information resource to support healthcare cost research and inform policy and practice. Med Care. 2009;47(7 Suppl 1):S44-50.

17. Agency for Healthcare Research and Quality. Medical Expenditure Panel Survey (MEPS): survey background. 2009. https://meps.ahrq.gov/about_ meps/survey_back.jsp. Accessed 12 Dec 2018.

18. Ware JE Jr, Kosinski M, Turner-Bowker D, et al. User's manual for the SF-12v2 health survey. 2nd ed. Lincoln, RI: QualityMetric; 2009.

19. Katz S, Ford AB, Moskowitz RW, Jackson BA, Jaffe MW. Studies of illness in the aged. The index of ADL: a standardized measure of biological and psychosocial function. JAMA. 1963;185:914-9.

20. Katz S, Downs TD, Cash HR, Grotz RC. Progress in development of the index of ADL. Gerontologist. 1970;10:20-30.

21. Lawton MP, Brody EM. Assessment of older people: self-maintaining and instrumental activities of daily living. Gerontologist. 1969;9(3):179-86.

22. Myers AM. The clinical Swiss army knife. Empirical evidence on the validity of IADL functional status measures. Med Care. 1992;30(5 Suppl):MS96-111.

23. Andersen RM. Revisiting the behavioral model and access to medical care: does it matter? J Health Soc Behav. 1995;36(1):1-10.

24. da Costa BR, Nüesch E, Kasteler R, et al. Oral or transdermal opioids for osteoarthritis of the knee or hip. Cochrane Database Syst Rev. 2014;(9): CD003115.

25. Krebs EE, Gravely A, Nugent S, et al. Effect of opioid vs nonopioid medications on pain-related function in patients with chronic back pain or hip or knee osteoarthritis pain: the SPACE randomized clinical trial. JAMA. 2018;319(9):872-82.

26. Webster LR. Opioid-induced constipation. Pain Med. 2015;16(Suppl 1):S16-21.

27. Smith H, Bruckenthal P. Implications of opioid analgesia for medically complicated patients. Drugs Aging. 2010;27(5):417-33.

28. Gore M, Tai KS, Sadosky A, et al. Clinical comorbidities, treatment patterns, and direct medical costs of patients with osteoarthritis in usual care: a retrospective claims database analysis. J Med Econ. 2011;14(4):497-507.

29. Sharma L, Cahue S, Song J, et al. Physical functioning over three years in knee osteoarthritis: role 
of psychosocial, local mechanical, and neuromuscular factors. Arthritis Rheum. 2003;48(12): 3359-70.

30. Mallen CD, Peat G, Thomas E, Lacey R, Croft P. Predicting poor functional outcome in communitydwelling older adults with knee pain: prognostic value of generic indicators. Ann Rheum Dis. 2007;66(11):1456-61.

31. van Dijk GM, Veenhof C, Spreeuwenberg P, et al. Prognosis of limitations in activities in osteoarthritis of the hip or knee: a 3-year cohort study. Arch Phys Med Rehabil. 2010;91(1):58-66.
32. Hayes CJ, Payakachat N, Li C. Evaluation of opioid use among patients with back disorders and arthritis. Qual Life Res. 2018;27(11):3021-35.

33. Hayes CJ, Li X, Li C, et al. Health-related quality of life among chronic opioid users, nonchronic opioid users, and nonopioid users with chronic noncancer pain. Health Serv Res. 2018;53(5):3329-49.

34. Brummett CM, Waljee JF, Goesling J, et al. New persistent opioid use after minor and major surgical procedures in US adults. JAMA Surg. 2017;152(6): e170504. 